\title{
Surface Alloying of the Casting in the Casting Mold
}

\author{
Rasulov Fuzuli, Babaev Agil \\ Faculty of Metallurgy, Azerbaijan Technical University, Baku, Azerbaijan \\ Email address: \\ resulovfr@gmail.com(R. Fuzuli),babaev@mail.ru(B. Agil)
}

\section{To cite this article:}

Rasulov Fuzuli, Babaev Agil. Surface Alloying of the Casting in the Casting Mold. Engineering and Applied Sciences. Vol. 3, No. 3, 2018, pp. 64-73. doi: 10.11648/j.eas.20180303.12

Received: July 16, 2018; Accepted: August 16, 2018; Published: August 29, 2018

\begin{abstract}
The article discusses the possibility of obtaining a composite coating on the surface of iron castings in the process of melt molding. As the composite powder mixture to the mold surface spreads used alloy powders and nickel-based lowalloyed white cast iron. Particle size distribution of these powders meet the following fractions: $+50-63 ;+63-100 ;+100-160$; $+160-200 ;+200-315 \mu \mathrm{m}$. It is established that a decrease in the thickness of the powder coating to $3-5 \mathrm{~mm}$ casting of cast iron into a mold with powder spread at $1420-1440^{\circ} \mathrm{C}$ and the dispersion of the powder particles $50 \ldots 100 \mu \mathrm{m}$ contribute to the formation of quality castings with a composite coating low-alloyed chrome-nickel white cast iron - gray cast iron and "nickel alloy CrNi80Si2B2 - gray cast iron". The increase in the casting temperature of cast iron from $1340^{\circ} \mathrm{C}$ to $1440^{\circ} \mathrm{C}$ increases the impregnation depth of the spread on the basis of the powder from low-alloyed chrome-nickel white cast iron and CrNi80Si2B2 by 3-4 $\mathrm{mm}$. It is found that by forming the composite coating on the surface of the mechanical properties and corrosion resistance of the castings are significantly increased compared to bare casting for the same composition. It has been established that the $\sigma_{\mathrm{B}}$ and $\mathrm{HB}$ of the material of the "CrNi80Si2B2 - gray cast iron" composite coating in comparison with cast iron increases in 1.86-1.94 and 1.20-1.24 times, wear resistance - by 1.5 times, and corrosion resistance in 43 times; for the material "low-alloyed chrome-nickel white cast iron - gray cast iron" these figures improve 1.44 times and 2.55 times, wear resistance 5.6 times and corrosion resistance 4.25 times. Optimum technological parameters for manufacturing of cast-iron castings with a composite coating "powder nickel alloy CrNi80Si2B2 - gray cast iron Class 20" with the raised wear resistance, corrosion resistance and durability are recommended. Technological processes have been developed for the manufacture of castings with a composite coating - type bushings and parts of cast-iron valves.
\end{abstract}

Keywords: Surface Alloying, Composite Coating, Casting, Mold, White Iron, Nickel Alloy

\section{Introduction}

In modern engineering and other industries, great attention is paid to finding effective and economic methods that would improve the operational durability of the working surfaces of cast parts operating under conditions of intense abrasion in active and aggressive environments. One such method is a method for surface alloying of a casting in a mold.

The effective methods are known that provide the necessary complex characteristics of the surface layer of castings by forming the structure and properties of the material in the process of impregnating the facing layer of the casting mold from the powder composite with liquid cast iron directly during casting [1-4].

The essence of this process, hardening of cast iron castings with a composite coating (CCC) is to apply a powder porous coating on the working surfaces of the mold and rods, drying, assembling and pouring the mold with a melt, the inner cavity of which corresponds to the configuration of the casting.

\section{Experimental Program}

\subsection{Aim and Objectives}

The aim of the work is to solve an important problem - to obtain the necessary complex of characteristics of the surface layer of castings by establishing the patterns of their formation and structure formation in the process of impregnating the putties from the powder composite with liquid cast iron directly in the mold;

1. study of the features of the casting process from gray cast iron of the pre-eutectic composition with liquid- 
glass coatings from low-alloyed chrome-nickel white cast iron, tin bronze, and nickel-base alloy;

2. development of the main technological parameters of the casting process of cast-iron CCC.

The following tasks were set in the work:

1. Investigation of the effect of granulometric compositions of powders and the density of a liquidglass binder on the technological properties of powder spread

2. Study of solidification kinetics of cast iron CCC and determine the main patterns of structure formation in the system "casting-compositional spread" under conditions of pouring into a sandy-clayey and centrifugal form;

3. Establishment of the main technological parameters of the impregnation process of powder spreading with liquid cast iron, which allows to selectively select powder composite composites to obtain quality CCC;

4. Manufacturing of cast iron castings - representatives with $\mathrm{CCC}$, carrying out an experimental industrial check of the technological process in production conditions.

\subsection{Method}

The studies and experiments presented in the present work were carried out on the physical models and laboratory installations of the Azerbaijan Technical University, as well as the conditions of the Baku Oil Machine Building Plant.

\section{Discussion of the Results}

In this article we present the results of studies of the effect of impregnation in the form of liquid cast iron of a layer of powder spreading from nickel-based alloys of composition (CrNi80Si2B2),\%: 0.2-0.3 C, 1.1-1.4 Si, 0.7-1.2 Mn, 07-0.8 $\mathrm{P}, 15-17 \mathrm{Cr}, 2.0 \mathrm{~B}, \leq 5 \mathrm{Fe}$, Ni- remaining and low-alloyed chrome-nickel white cast iron (LACrNiWCI) composition, \%: 2.64-4.07 C, 0.58- 0.56 Si, 0.70-0.74 Mn, 0.08 P, 0.05 S, 0.36 $\mathrm{Ni}, 1.57-1.55 \mathrm{Cr}, \mathrm{Fe}$ is the rest on the formation of the structure and properties of the metal castings from gray cast iron and a composite coating (CC), as well as the results of the development of the technological process for the production of high-quality cast iron $\mathrm{CCC}$, produced in single and trobezhnyh forms.

For research castings from gray cast iron of class 20 and a powder and from alloys LACrNiWCI, CrNi80Si2B2 were used.

The granulometric composition of the powders corresponded to the fractions: $+50-63 ;+63-100 ;+100-160$; 160-200 and 200-315 $\mu \mathrm{m}$.

To this end, we have investigated compacts with fine powders $(30-50 \mu \mathrm{m})$, medium $(50-100 \mu \mathrm{m})$ and large $(>100$ $\mu \mathrm{m})$ sizes and liquid glass with densities $\rho=1.29 ; 1.37$ and $1.42 \mathrm{~g} / \mathrm{cm}^{3}$.

Dependences between the depth of impregnation of the melt in the powder spread, the density of the metal CC, and the casting temperature of cast iron with different fractional and chemical compositions of the powder particles are investigated.

Increasing the casting temperature of cast iron from 1300 to $1450^{\circ} \mathrm{C}$ can achieve an increase in the rate of its filtration by 2-3 times [5]. It was established that an increase in the thickness of powder spreads from 5 to 10 and $15 \mathrm{~mm}$ resulted in a decrease in the content of the elements of the CrNi80Si2B2 alloy in thin interparticle layers of cast iron; namely: by nickel - from $9.62 \%$ to 4.38 and $6.51 \%$; on chromium - from $2.19 \%$ to 0.25 and $1.31 \%$ and on silicon from $2.22 \%$ and 0.34 and $0.36 \%$. Whereas the content of phosphorus in cast iron impregnated in porous putty increases from $0.42 \%$ to $0.51-0.61$. In all cases of casting with impregnation of powder coating, the metal of the contact zone of the base iron with the $\mathrm{CC}$ is significantly more saturated with $\mathrm{Ni}, \mathrm{Cr}$, and $\mathrm{P}$ than in the peripheral zone.

The main factors influencing the operational characteristics of the CCC are: firstly, the regulated content of alloying elements in the alloy powder, which determines the phase composition and volume fraction of composite compounds; secondly, the technological regimes of impregnating the powder paste with liquid iron to obtain the required level of properties, which are determined by the morphology of the phase components [6]. The study was carried out with plaster of LACrNiWCI thickness 3, 5 and 10 $\mathrm{mm}$.

It is established that the increase in hardness in a $5 \mathrm{~mm}$ thick CC material based on the LACrNiWCI powder from $4.07 \% \mathrm{C}$ is higher than for those of the same powder with $2.64 \% \mathrm{C}$, which indicates a significant effect of the carbon content on hardness (Figure 1). There is a slight increase in hardness in the transition zone CC-gray cast iron in the direction from the surface of the CC to the casting. This is due to the fact that in the warmer deep layers of powder spreading from the LACrNiWCI during the filtration by liquid cast iron, the particles of the high-carbon phasecementite dissolve. When the powder contains $2.64 \%$ of carbon in the near-boundary zone of the $\mathrm{CC}$ with freshly liquid cast iron, carbon depletion occurs in the powder from the LACrNiWCI, and $4.07 \% \mathrm{C}$ is added to the powder in the powder. Alternatively, liquid iron is saturated with carbon. This determines its crystallization by structural-white, which determines the increase in the hardness of the bleached casting layer.

With an increase in the thickness of the putty from $3 \mathrm{~mm}$ to $10 \mathrm{~mm}$, a noticeable difference in the hardness of the metal of the surface zone of the $\mathrm{CC}$ is observed at a carbon content of up to $4.07 \% \mathrm{C}$ in the powder iron composition (Figure 1). This is explained by the fact that, with a higher carbon content, the change in the structure caused by impregnation of the melt plays a significant role because of the increase in the cementite phase and the decrease in the volume of the viscous phase, austenite. Increasing the thickness of the putty from 3 to 5 and $10 \mathrm{~mm}$ increases the hardness of cast iron (Class 20) castings (wall thickness $40 \mathrm{~mm}$ ) from 1780 to 2050 and $2150 \mathrm{MPa}$, respectively.

In order to exclude the influence of the casting crust, a 
metal layer $0.2 \mathrm{~mm}$ thick was removed from the samples by grinding before measuring the hardness of the surface of the $\mathrm{CC}$ castings. Samples from the $\mathrm{CC}$ had a thickness of 3,5 and $10 \mathrm{~mm}$, formed from LACrNiWCI spreads when impregnated with liquid cast iron. With an increase in the thickness of powder spreads from 3 to $5 \mathrm{~mm}$ and $10 \mathrm{~mm}$, as well as an increase in the carbon content in the $\mathrm{CC}$, the hardness of the samples increases. With a $2.64 \%$ carbon content in powdered cast iron, the hardness of the $\mathrm{CC}$ with a thickness of 3,5 and $10 \mathrm{~mm}$ varies within $33 \ldots . .37 \mathrm{HRC}$. Other things being equal, with a CC thickness of $3 \mathrm{~mm}$ with an increase in the carbon content of the powder composition from $3.17,3.61$ to $4.07 \%$, the hardness of the material base is reduced; the microstructure of the surface zone of the $\mathrm{CC}$ corresponds to the structure of the half-cast iron, which is the result of partial graphitization of the powder particles. Increasing the thickness of the powder spread to 5 and 10 $\mathrm{mm}$ increases the hardness of the $\mathrm{CC}$ material; moreover, the higher the carbon content in the cast iron of the powder, the higher is its value: at $3.17 \% \mathrm{C}$ by $4-7$ and $6-8 \mathrm{HRC}$, at $3.61 \%$
$\mathrm{C}$ at $6-12$ and 12-15 HRC and at 4.07\% $\mathrm{C}$ at 22-24 HRC, respectively.

It was found that casting of cast iron with a relatively low superheat $\left(60-80^{\circ} \mathrm{C}\right)$ does not allow increasing the depth of impregnation of liquid iron in powder spread (no more than $1.5-2 \mathrm{~mm}$ ). Pouring cast iron with superheating at $120-150^{\circ} \mathrm{C}$ allows to increase the time interval between the beginning and the end of solidification to 5-6 s, which ensures the production of CC with a thickness of up to $12-14 \mathrm{~mm}$ with a sufficiently accurate outline and dense structure.

The test results showed that the mechanical properties of cast iron CCC (with a wall thickness of $40 \mathrm{~mm}$ ) with a thickness of 10-12 mm CC is much higher than that of cast iron, hardened without coating. It was found that the composition and dispersity of the powder of the putty have almost no effect on the strength properties of cast iron, and the main influence is exerted by the magnitude of the plates of graphite inclusions. It is found that for iron of CCC with an almost equal hardness the tensile strength is $30-36 \mathrm{MPa}$ more than cast iron without it.

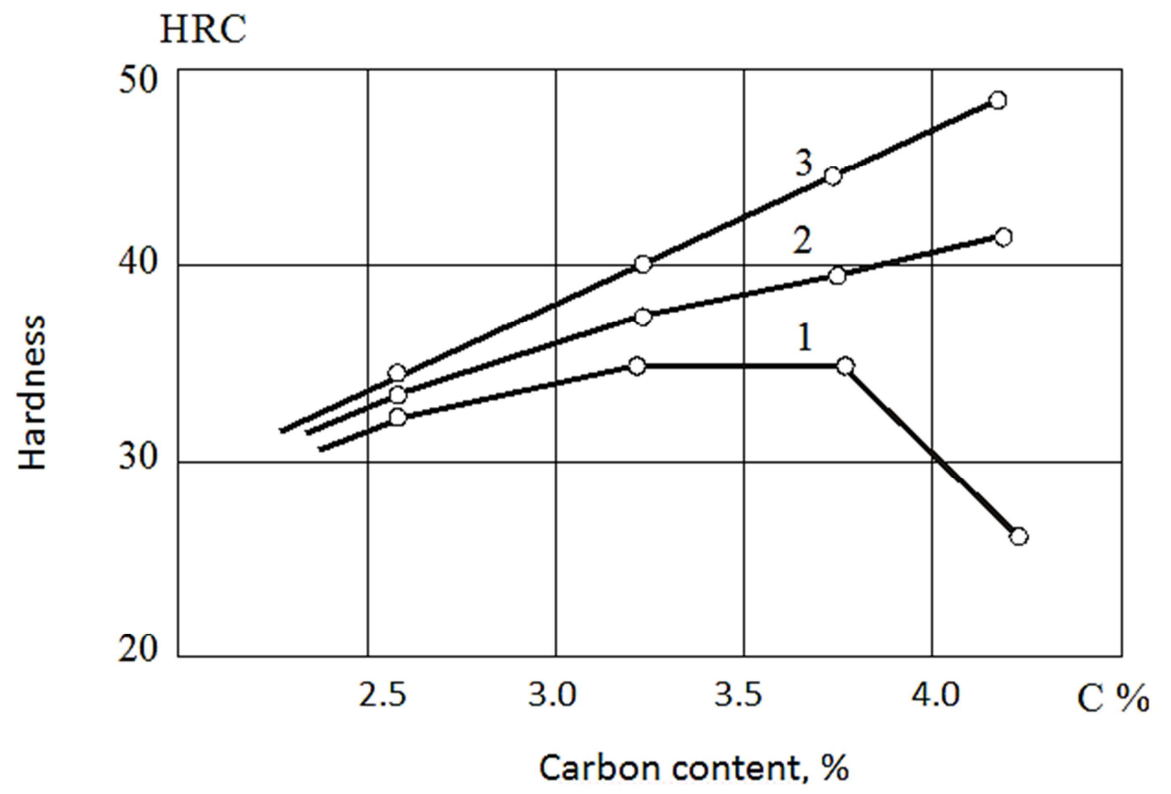

Figure 1. Extensibility of the submerged thrustability of the oxygen in the powder and the thicknesses of the CC: 1, 2 and 3 - the thickness of prayers 3, 5 and $10 \mathrm{~mm}$, correspondingly.

The results of testing the $\mathrm{CC}$ material of the castings "LACrNiWCI - gray cast iron" and "CrNi80Si2B2-gray cast iron" showed that the mechanical properties $\left(\sigma_{B}\right.$ and $\left.\mathrm{HB}\right)$ are significantly higher than for cast iron castings without coating (Figure 2).

The maximum strength properties have the material of the "CrNi80Si2B2-gray cast iron" CC, while the $\sigma_{B}$ and $\mathrm{HB}$ of the material of the $C C$ are increased by $1.86-1.94$ and 1.20 1.24 times, respectively, compared with the cast iron.

The results of testing the materials of the "LACrNiWCI - gray cast iron" CC showed that the chemical composition of powder porous spread significantly affects their strength properties and in all cases, they are slightly higher than that of cast iron without castings. Thus, the ultimate strength of the material of the "LACrNiWCI - gray cast iron" CC is higher by 100-110 MPa than in gray cast iron.

The effect of impregnation conditions and the thickness of the putty on the hardness of the "CrNi80Si2B2-gray cast iron" CC is shown in Figure 3. 

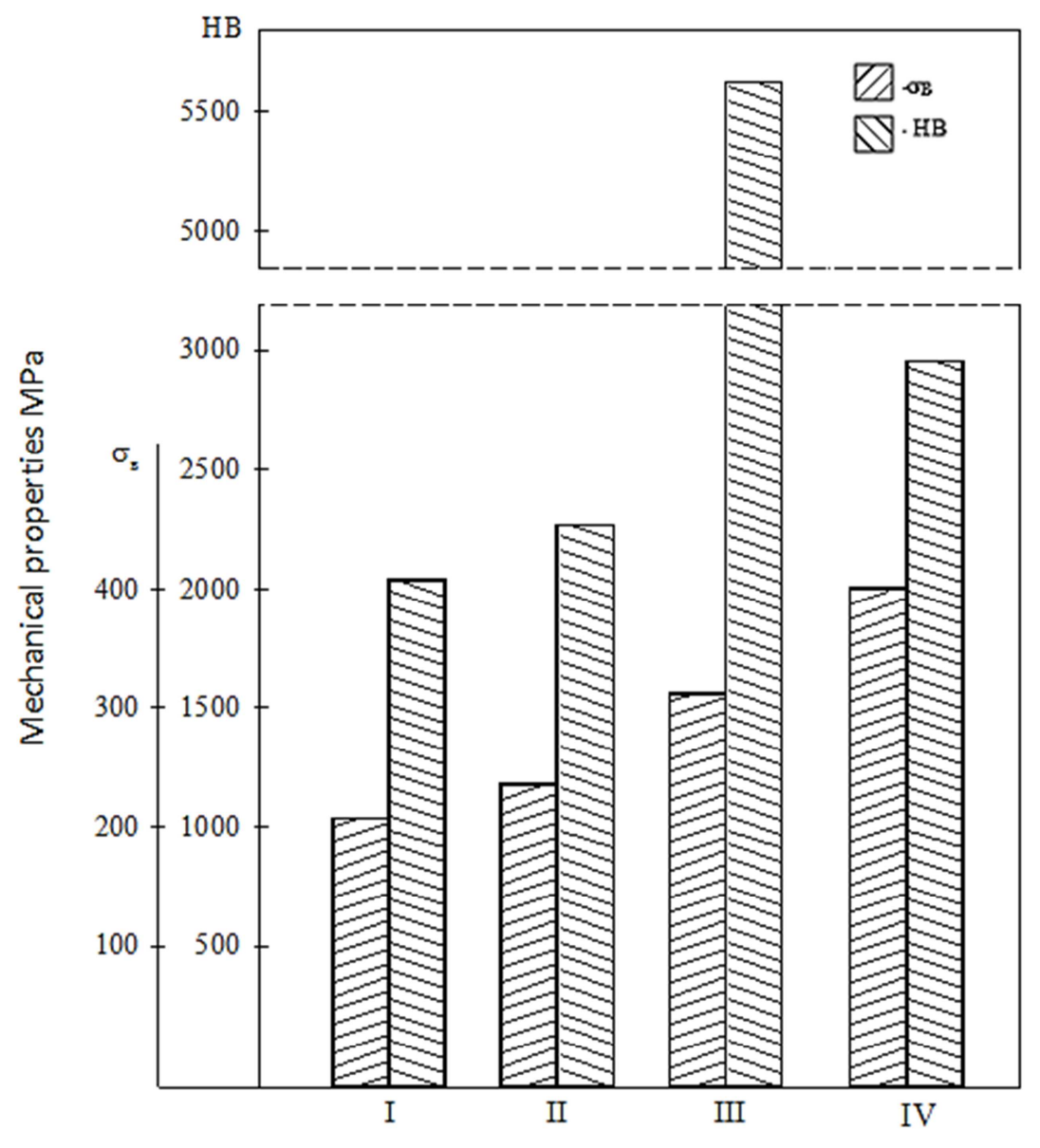

Type of cast iron and composite casting coating

Figure 2. Comparative mechanical properties of cast iron and CC castings: I - cast iron without coating; II - the same with CC gray cast iron; III - CC LACrNiWCI -gray cast iron; IV-CC CrNi80Si2B2- gray cast iron.

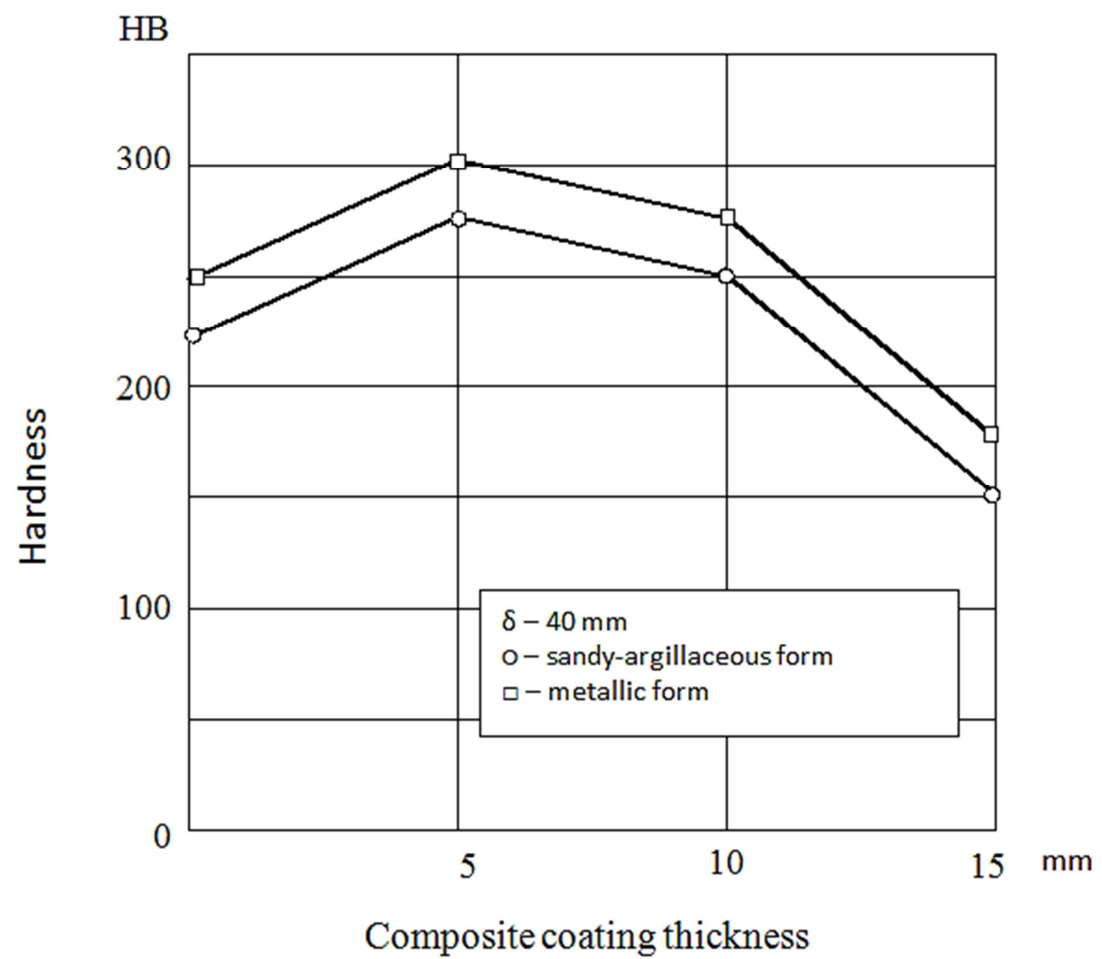

Figure 3. Influence of the impregnation conditions and the thickness of the putty on the hardness CrNi80Si2B2 - gray cast iron'CC. 
The resistance of the anti-abrasion specimens was tested in pairs with a diamond wheel while rotating on an MT-66 machine (slip speed $0.5 \mathrm{~m} / \mathrm{s}$, load $48 \mathrm{~N}$ ), as well as at a constant pressure of $0.015 \mathrm{~N} / \mathrm{m}^{2}$ and a slip velocity of 1.25 $\mathrm{m} / \mathrm{s}$.

The wear resistance of samples cut from the cast iron wall was compared with a structure consisting of a perlite-ferritic metal base with lamellar graphite; and with $\mathrm{CC}$ on the basis of CrNi80Si2B2- gray cast iron, BrO10S1.5C0.5-gray cast iron, LACrNiWCI powder - gray cast iron, white cast iron powder $+10 \%$ FeB-cast iron and austenitic nickel-chromium pig iron of the NIREZIST type (monometall) (Figure 4).

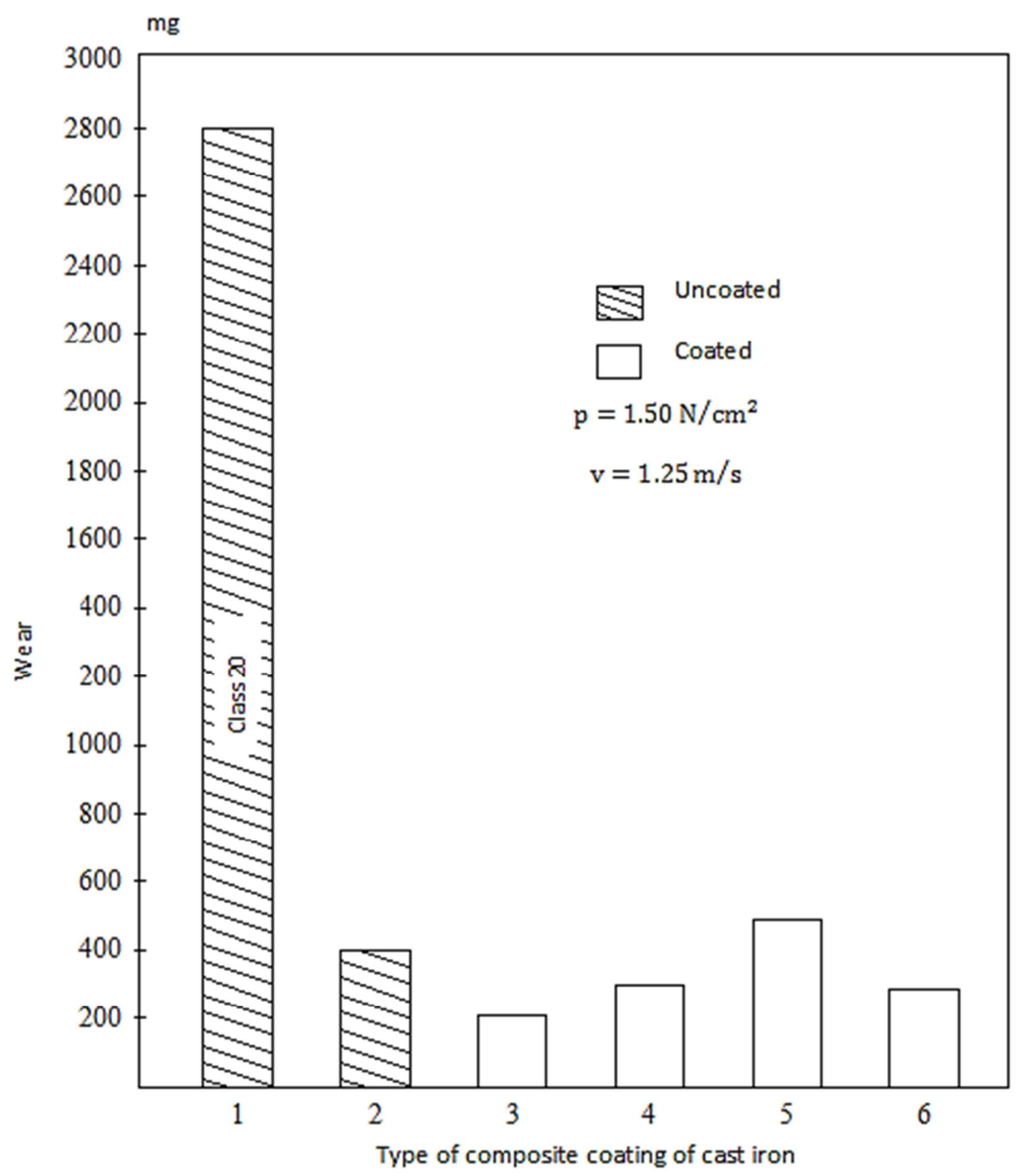

Figure 4. Comparative wear resistance of CC cast iron: 1 - gray cast iron class 20; 2 - NIREZIST; 3 - CrNi80Si2B2 - gray cast iron; 4 - BrO10S1.5C0.5-gray cast iron; 5 - LACrNiWCI-gray cast iron; 6 - LACrNiWCI + 10\% FeB-gray cast iron.

It is known that the wear resistance of materials depends on the hardness and the wear rate decreases with its growth [7].

The anti-abrasion speed of the samples paired with the diamond wheel was evaluated by rotation on an MT-66 machine (sliding speed $0.5 \mathrm{~m} / \mathrm{s}$, load $48 \mathrm{~N}$ ). The results of testing specimens cut from the reverse surface zone of castings without $\mathrm{CC}$ showed a high wear rate.

As the thickness of the powder coating increases, the resistance of the metal $\mathrm{CC}$ to the wear resistance increases. Thus, increasing the thickness of the coating from 3 to 5 and $10 \mathrm{~mm}$ helps to reduce the wear rate from $783 \mu \mathrm{m} / \mathrm{min}$ to 741 and $718 \mu \mathrm{m} / \mathrm{min}$. respectively.

It follows from figure 5 that the $\mathrm{CC}$ obtained by impregnating a $5 \mathrm{~mm}$ thick powder spread is more stable. The upper and average values of wear resistance of the CC material correspond to samples taken from castings filled at a temperature above $1400^{\circ} \mathrm{C}$.

As can be seen, the wear rate is affected both by the carbon content of the LACrNiWCI powder and by the thickness of the spread applied to the working surface of the casting sandy-clay mold.

Thus, at a powder thickness of $3 \mathrm{~mm}$ with an increase in the carbon content of powdered, LACrNiWCI from 2.64 to 3.61 and $4.07 \%$, the wear rate increases by 87 and $25 \mu \mathrm{m} / \mathrm{min}$ (Figure 5, samples 1, 2 and 3 ). This is due to the graphitization of powder particles over the entire thickness of the spread, which contributes to an increase in the number of graphite inclusions and a decrease in hardness with an increase in the carbon content [8]. The increase in the thickness of the powder coating and the carbon content of the LACrNiWCI impregnation differently affect the wear resistance of the $\mathrm{CC}$ material of the castings. With a coating thickness of $5 \mathrm{~mm}$ and $10 \mathrm{~mm}$, an increase in the carbon content to 3.61 and $4.07 \%$ reduces the wear rate of the samples at 68 and $93 \mu \mathrm{m} / \mathrm{min}$ and 52 and $70 \mu \mathrm{m} / \mathrm{min}$, respectively (Figure 5, samples 4, 5, 6 and 7, 8, 9). Comparison of the results of the study of the change in the 
hardness of the surface zones of $\mathrm{CC}$ and the wear rate of samples from coatings with different carbon contents and thicknesses showed that, despite the increase in their hardness, with an increase in the carbon content in powdered iron (Figure 1), the wear rate of samples with a coating thickness of $5 \mathrm{~mm}$ less than at $10 \mathrm{~mm}$, the best indices in samples 5 and 6 . It is obvious that a slight increase in the wear rate in samples 7,8 and 9 is due to the fact that at the time of infiltration into the periphery th zone spreads the powder at the interface between the particles of the powder and the liquid phase diffusion processes are defective, and that the porosity of the material is much greater. Under the pressure of the counterbody on the microscopic sections of the actual contact of the friction surfaces, the unmelted powder particles are chipped and the metal mass is chipped at the locations of the micropores in the surface layer of the 10$\mathrm{mm}$-thick CC. Therefore, the wear process proceeds with the chipping of some unmelted particles of sample material made from a $10 \mathrm{~mm}$ thick $\mathrm{CC}$ sheet.

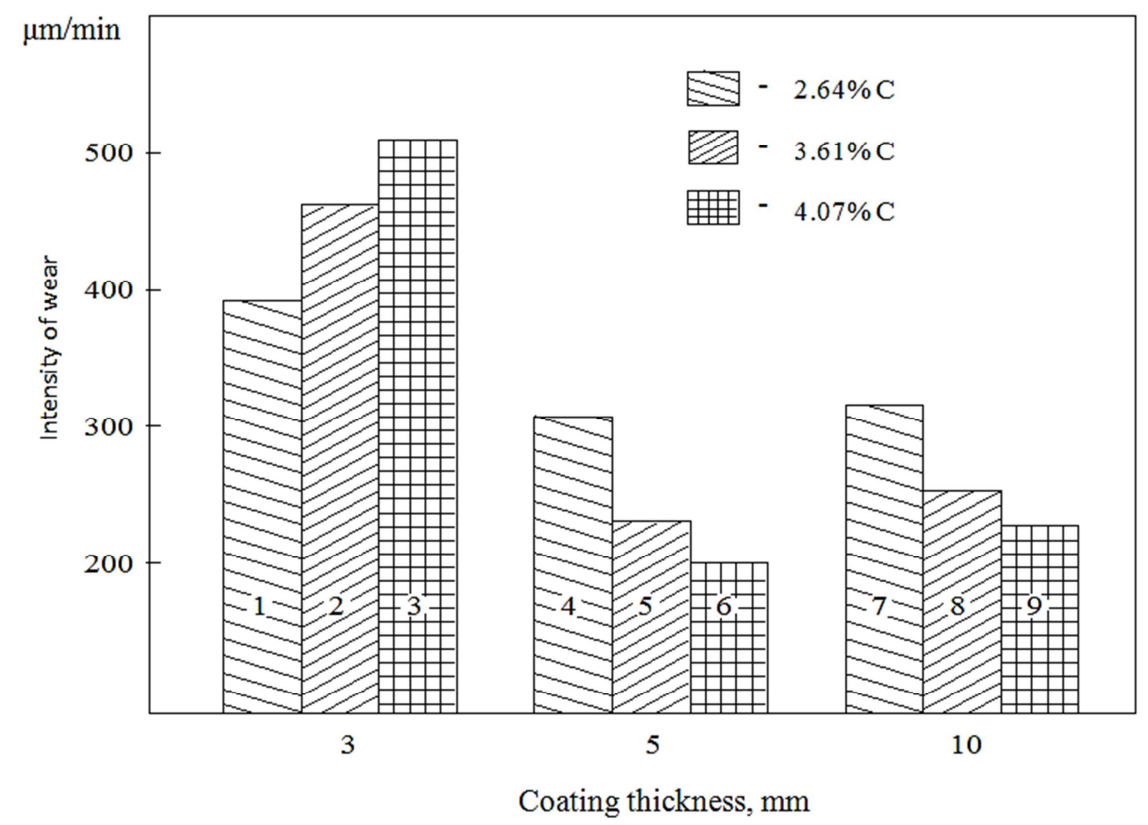

Figure 5. Dependence of the wear rate of the material LACrNiWCI-gray cast iron"CC on the carbon content and its thickness: 1 , 4 and 7 - with a content of $2.64 \% \mathrm{C} ; 2,5$ and 8 - the same - 3.61\% C; 3, 6 and 9 - the same - $4.07 \% \mathrm{C}$

Investigation of wear processes of cast iron $\mathrm{CC}$ on samples in laboratory conditions and analysis of literature data allow us to conclude that the method of obtaining CC in castings impregnated with powder composites in the process of casting directly in the mold can be most widely used in the fight against wear of machine parts. This technology does not complicate the process of making castings and does not require large material costs.

A study of the rate of wear of the samples, depending on the conditions for the formation of $\mathrm{CC}$ castings, the composition of the material of powder composites, and the slip velocity $(1.25 \mathrm{~m} / \mathrm{sec})$ was carried out under laboratory conditions.

In the process of friction, a reference sample of the CC specimen from austenitic cast iron of the NIREZIST type on its surface breaks out the particles of the composite metal, some of which adhere to the conjugate surface, and the rest are wearing products.

The destruction of the friction surface of a specimen from CC "CrNi80Si2B2-gray cast iron" has a local character. It occurs according to the cementite-ledeburite phases of cast iron ligament (metal bonds), and not by much less durable metal CC brand CrNi80Si2B2, as the cementite-ledeburite phase has a lower impact strength.
Comparison of the relative wear resistance of the $\mathrm{CC}$ specimens from "CrNi80Si2B2- gray cast iron" and "LACrNiWCI-gray cast iron" with samples from monocastings of highly wear-resistant bulk doped austenitic nickel-chromium-cast iron (NIREZIST) showed that wear of the CC materials "CrNi80Si2B2- gray cast iron" and "LACrNiWCI-gray cast iron" 3.86 and 2.5 times less than in the reference sample made of cast iron NIREZIST; under analogous conditions of wear, the material from the "LACrNiWCI-gray cast iron" CC material is slightly inferior in terms of wear resistance (0.94) to the "NIREZIST" monogauge indicator.

The structure of the $\mathrm{CC}$, which reduces wear, consists of powder particles with inclusions of chromium carbide, nickel boride and iron. The hardness of this layer is up to 1000 units by Vickers.

Thus, carried out studies of the wear resistance of cast iron samples with CC allowed us to establish that this method of obtaining cast iron CCC can be recommended for increasing the durability of parts working under friction conditions. The use of wear-resistant $\mathrm{CC}$ on gray cast iron castings will not only increase the service life of reinforcement parts, but also recommend the replacement of high-chromium-nickel austenitic cast iron such as NIREZIST (with a chemical 
composition of $18-36 \% \mathrm{Ni}, 1-4 \% \mathrm{Cr}$ and $3 \% \mathrm{Cu}$ ) and steels of the type $12 \mathrm{Cr} 18 \mathrm{Ni} 9 \mathrm{Ti}$ casting.

The possibility of increasing the corrosion resistance of the working surface of reinforcing cast iron parts by the method of impregnation in a mold with powder spreads from LACrNiWCI and CrNi80Si2B2 alloys was investigated.

Tests for corrosion resistance of the $\mathrm{CC}$ "powder from LACrNiWCI - gray cast iron" were carried out according to the accepted method in media: sea water, 3\% aqueous solution of sodium hydroxide, $10 \%$ solutions of hydrochloric, nitric and sulfuric acids. At a temperature of $20^{\circ} \mathrm{C}$ for 110 hours, four samples with dimensions of 40x10x8 mm were tested, which were cut from a $10 \mathrm{~mm}$ thick CC panel and a mid-wall $(40 \mathrm{~mm})$ of experimental cast iron. Powder spread consists of LACrNiWCI-gray cast iron. Samples before the test were ground, degreased, washed in alcohol and weighed, and after tests in corrosive media they were cleaned of corrosion products, washed in alcohol and weighed again. The results of the tests are given in Table 1.

Table 1. Corrosion resistance of the CC and the base of the casting wall (Impregnation temperature $1380{ }^{\circ} \mathrm{C}$ ).

\begin{tabular}{|c|c|c|c|c|c|c|}
\hline \multirow{2}{*}{ No. } & \multirow{2}{*}{ Corrosive environment } & \multirow{2}{*}{ Concentration, $\%$} & \multirow{2}{*}{ Temperature, ${ }^{\circ} \mathrm{C}$} & \multicolumn{2}{|c|}{ Corrosion rate of samples $\mathrm{g} / \mathrm{m}^{2} \mathrm{~h}$} & \multirow{2}{*}{$\begin{array}{l}\text { Increased corrosion } \\
\text { resistance, fold. }\end{array}$} \\
\hline & & & & Cast iron foundations & Composite coating & \\
\hline 1. & $\begin{array}{l}\text { Aqueous solution of } \\
\text { sodium hydroxide }\end{array}$ & 3 & 20 & 4.82 & 0.71 & 6.78 \\
\hline 2. & Sea water & - & $20-30$ & 0.23 & 0.04 & 5.75 \\
\hline 3. & Hydrochloric acid & 10 & 20 & 25.47 & 5.98 & 4.25 \\
\hline 4. & Nitric acid & 10 & 20 & 63.18 & 29.12 & 2.16 \\
\hline 5. & Sulfuric acid & 10 & 20 & 29.25 & 6.10 & 4.79 \\
\hline
\end{tabular}

Being in the iron-carbon alloy of the powder, chromium partially enters the solid solution and partially forms complex carbides of the type $(\mathrm{Fe}, \mathrm{Cr})_{3} \mathrm{C}$ with carbon [9]. When the content of cast iron is $2.64 \ldots 4.07 \%$ carbon, in addition to complex carbides, a purely cementite phase is formed, the potential of which differs from the potential of the solid solution, which contributes to the formation of micro-pairs and the destruction of the $\mathrm{CC}$ of the casting. Consequently, the properties of the material of the CC "LACrNiWCI-gray cast iron" are largely determined not only by the amount of chromium $(\sim 1.5 \%)$ and nickel $(0.46 \%)$ but also by the carbon content in them.

The results of the tests showed that the $\mathrm{CC}$, whose structure consists of unmelted powder particles and interparticle interlayers of cementite-ledeburite constituents, anticorrosive properties in sea water, low-concentration acids and strong electrolytes significantly increase in comparison with the cast iron base. The upper values of the corrosion resistance of the composite material correspond to the samples tested in media of aqueous sodium hydroxide $(6.78$ times), sea water (5.75 times), 10\% hydrochloric, nitric and sulfuric acids (4.25, 2.16 and 4.79 times, respectively).

Figure 6 shows the average values of the corrosion resistance test results for five samples from CC castings obtained by impregnation with powder spread, when casting cast iron with a temperature of $1380^{\circ}$ and $1440^{\circ} \mathrm{C}$.

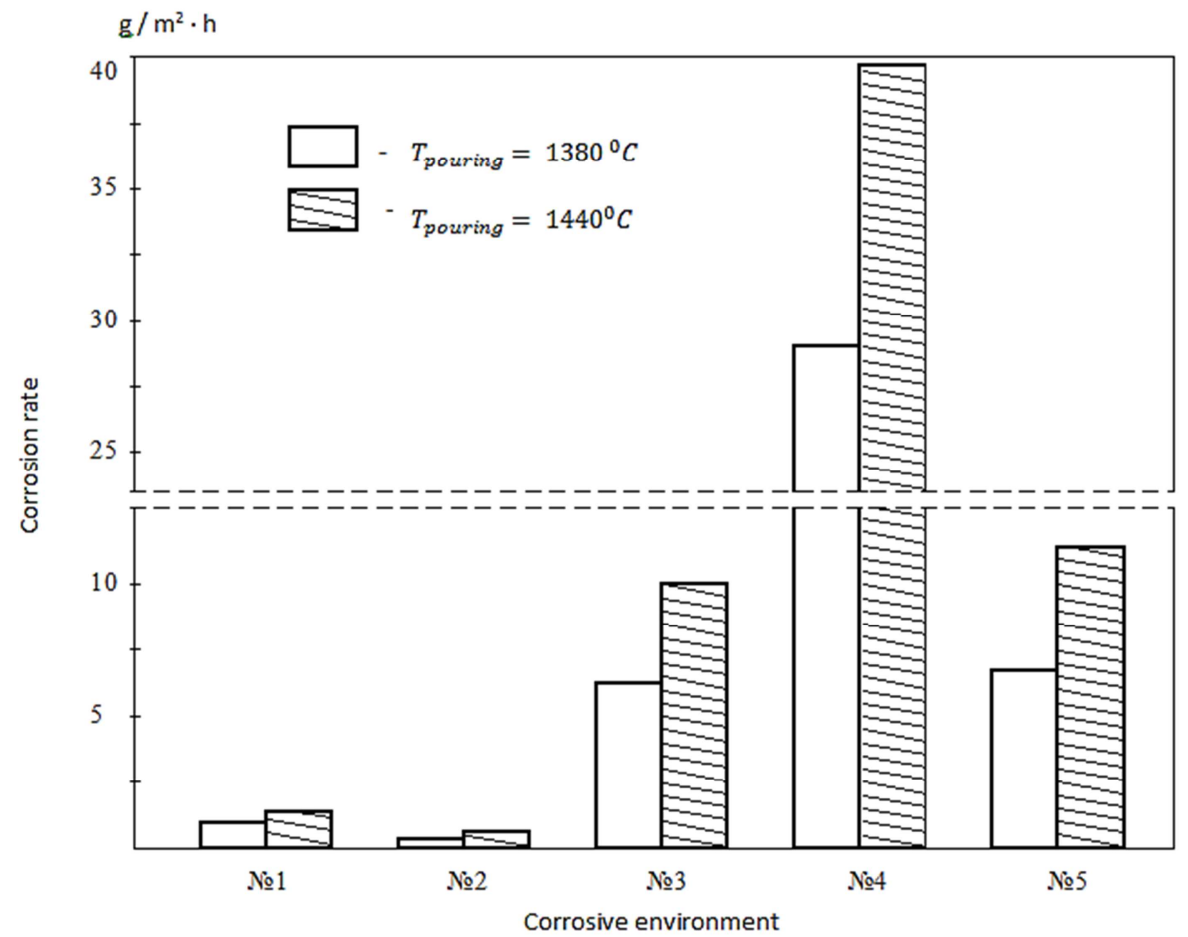

Figure 6. The change in the corrosion resistance of the material LACrNiWCI - gray cast iron"CC depending on the impregnation temperature: 1, 2, 3, 4 and 5 - the samples tested in the media: $3 \%$ aqueous solution of caustic sodium, sea water and 10\%, 10\% nitrogen and 10\% sulfuric acid, respectively. 
The best results of corrosion resistance are obtained in impregnated samples with casting liquid iron at a temperature of $1380^{\circ} \mathrm{C}$.

It is known that in a powder of the alloy, CrNi80Si2B2chromium is an important nickel alloying element; while nickel also has the ability to dissolve in large quantities many elements, such as iron and silicon. The main advantage of nickel-chromium alloys is their high corrosion resistance in a wide range of oxidation and reduction media. Properties of nickel significantly depend on the content of impurities such as carbon, sulfur, phosphorus and oxygen in it.

When the thickness of the CC casting "CrNi80Si2B2-gray cast iron" 5 and $10 \mathrm{~mm}$ corrosion damage spreads evenly from the peripheral surface into the interior of the sample. In a sample of a $5 \mathrm{~mm}$ thick $\mathrm{CC}$, corrosion is local in nature and localized in the areas of the surface where it is in contact with the fine cemented-iron-cementite phase of the cast iron and the sintered mass of the powder composite of the alloy CrNi80Si2B2 in the form of single points or spots [10].

Since corrosion damage always starts from the surface, there is no need to increase the thickness of the $\mathrm{CC}$ in the castings.

Tests for corrosion resistance were carried out at room temperature in open cups according to the generally accepted procedure. The test time varied from 5 to 100 hours. Corrosion resistance was evaluated by loss of mass per unit of initial surface area of the samples.

It is known that in dilute aqueous solutions of hydrochloric and sulfuric acids, iron-carbon alloys are unstable. The exception is high-silica cast iron and chromium-nickel steels. The latter, due to their high hardness, brittleness and sensitivity to temperature fluctuations, have very limited application [11].

From the "CrNi80Si2B2 - gray cast iron" CC with a thickness of 3,5 and $10 \mathrm{~mm}$, coatings of 5 and $10 \mathrm{~mm}$ thick have the greatest corrosion resistance in dilute sulfuric and hydrochloric acid media. In aqueous solutions of sulfuric acid with a concentration of 15 and $20 \%$, the corrosion rate of the CC material decreases by an average of 15 times in comparison with the base cast iron, and an average of 43 times in aqueous solutions of hydrochloric acid in the above concentrations.

Comparative data on the corrosion resistance of metal $\mathrm{CC}$ "CrNi80Si2B2- gray cast iron" castings of different thicknesses to the action of dilute sulfuric and hydrochloric acids is given in Table 2 .

Table 2. Change in the corrosion resistance of cast iron depending on the thickness of the CrNi80Si2B2-class 20'CC.

\begin{tabular}{|c|c|c|c|c|c|c|}
\hline \multirow{3}{*}{ Material } & \multicolumn{6}{|c|}{ Corrosion rate, $\mathrm{g} / \mathrm{m}^{2} \cdot \mathrm{h}$} \\
\hline & \multicolumn{6}{|c|}{ The concentration of sulfuric acid at $20^{\circ} \mathrm{C}, \%$} \\
\hline & 1.0 & 3.0 & 5.0 & 10.0 & 15 & 20 \\
\hline Class 20/40 & 48.4 & 106.6 & 122.3 & 98.10 & 72.85 & 43.05 \\
\hline CrNi80Si2B2/3- Class 20 & 0.031 & 0.104 & 0.172 & 0.217 & 0.111 & 0.084 \\
\hline CrNi80Si2B2/5- Class 20 & 0.001 & 0.025 & 0.033 & 0.093 & 0.006 & 0.005 \\
\hline CrNi80Si2B2/10- Class 20 & 0.007 & 0.032 & 0.083 & 0.106 & 0.002 & 0.008 \\
\hline Cast iron NIREZIST & 0.001 & 0.003 & 0.47 & 1.831 & 1.455 & 1.481 \\
\hline Class 20/40 & 82.263 & 199.141 & 225.465 & 310.442 & 277.373 & 301.106 \\
\hline CrNi80Si2B2/3- Class 20 & 2.099 & 2.644 & 2.956 & 3.256 & 3.484 & 3.678 \\
\hline CrNi80Si2B2/5- Class 20 & 1.808 & 2.038 & 2.077 & 2.196 & 2.223 & 2.424 \\
\hline CrNi80Si2B2/10- Class 20 & 1.970 & 2.365 & 2.572 & 2.812 & 2.971 & 2.910 \\
\hline Cast iron NIREZIST & 3.717 & 4.892 & 4.776 & 4.611 & 4.705 & 4.601 \\
\hline
\end{tabular}

The results of the corrosion resistance testing of the materials "CrNi80Si2B2- gray cast iron" CC showed that, from the economic point of view, it is advantageous to obtain the CCC with the smallest thickness $(\sim 5 \mathrm{~mm})$ of the CC, which makes it possible to reduce the consumption of the deficit CrNi80Si2B2 powder on the product. However, one should take into account the fact that when the thickness of the putty is reduced, the content of cementite-ledeburite components increases in the material of the $0 \mathrm{CC}$.

It is established that with increasing casting temperature of cast iron from 1360 to $1440^{\circ} \mathrm{C}$ the depth increases, the fraction of insoluble powder particles in the structure of CC "CrNi80Si2B2-gray cast iron" decreases from $68-65 \%$ to 45 $48 \%$, which is associated with an increase in the solubility of powder particles in putties in cast iron ligaments. In this case, the porosity of the $\mathrm{CC}$ unit decreases by $0.91-1.33 \%$ and the interlayer between the undissolved particles increases from
$0.10-0.12 \mathrm{~mm}$ to $0.22-0.28 \mathrm{~mm}$, which contributes to the improvement of the strength properties of the $\mathrm{CC}$ material of the cast iron.

It has been established that the $\sigma_{B}$ and $\mathrm{HB}$ of the material of the "CrNi80Si2B2 - gray cast iron" CC in comparison with cast iron increases in 1.86-1.94 and 1.20-1.24 times, wear resistance - by 1.5 times, and corrosion resistance in 43 times; for the material "LACrNiWCI-gray cast iron" these figures improve 1.44 times and 2.55 times, wear resistance 5.6 times and corrosion resistance 4.25 times.

Recommended optimal technological parameters for the manufacture of cast iron CCC "powder nickel alloy CrNi80Si2B2 - gray cast iron class 20" with increased wear resistance, corrosion resistance and strength. Technological processes have been developed for the manufacture of CCCtype bushings and parts of cast-iron valves (Figure 7). 


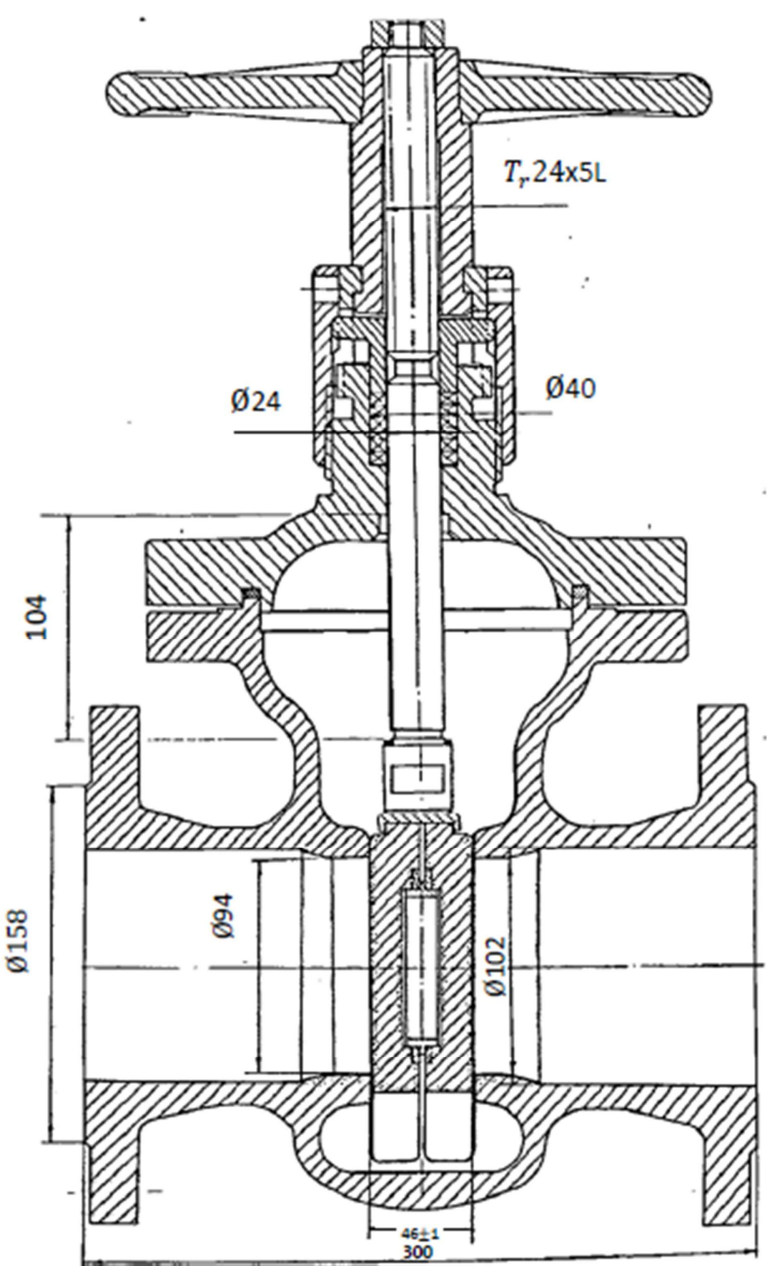

a)

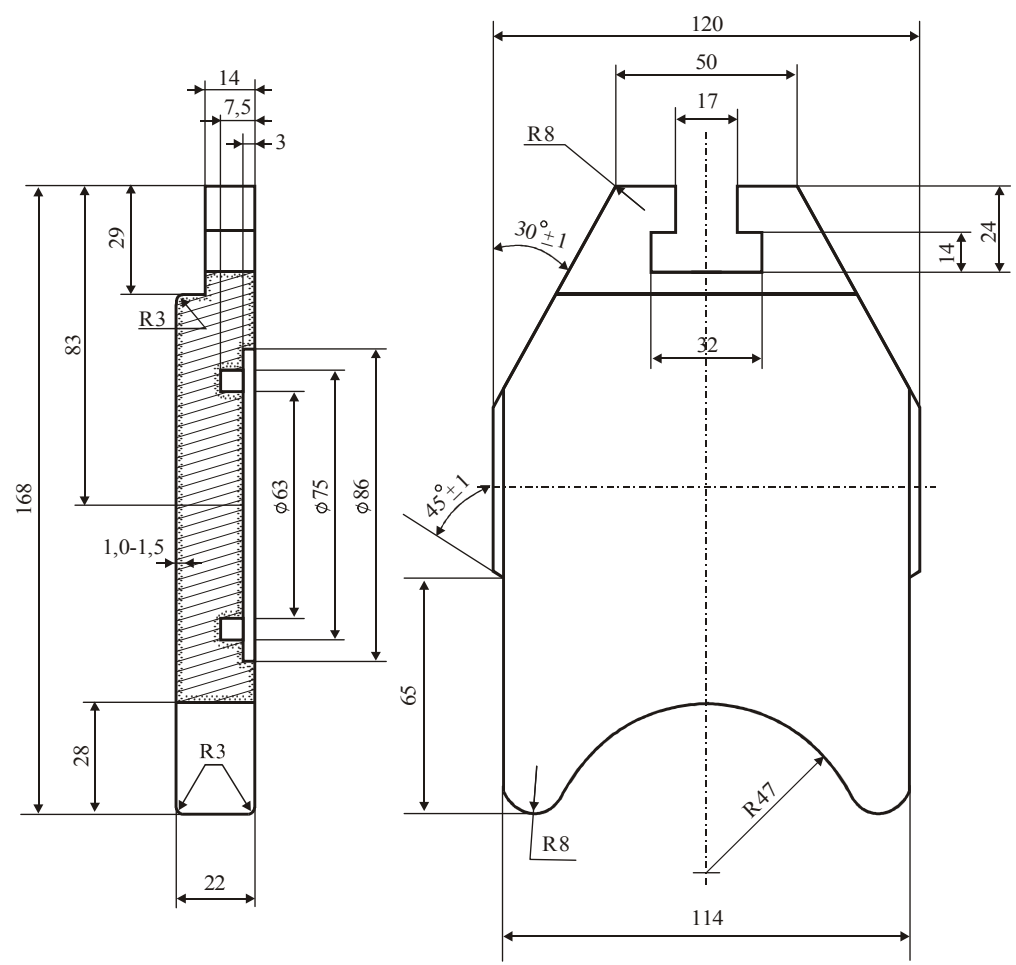

b)

Figure 7. Assembly drawing of the AZL valve, DN 100 (a) and cast iron disk with composite coating of the AZL valve (b). 


\section{Conclusion}

Investigations established technological modes of manufacturing cast-iron CCC type powder nickel alloy "CrNi80Si2B2-gray cast iron" and "LACrNiWCI-gray cast iron " with increased strength, hardness, wear resistance and corrosion resistance. In this case, the best surface properties of the castings are provided with a coating thickness of 5 $\mathrm{mm}$. When using LACrNiWCI as a coating, a high hardness of the casting surface is achieved, corresponding to the hardness of the hardened cast iron.

\section{References}

[1] Bukhtoyarova V. N. An overview of the method for joint spraying and hardening of coatings. Voronezh. Techn. Academy, Voronezh, 2001, 9 p.

[2] Rasulov F. R. Effects of technological factors on the impregnability of powder spread. Baku, AzTU, Doctrine of Recording, 2010, p. 70-75.

[3] Study of the possibility of continuous casting of bimetallic components in the condition of direct connection of metals in a liquid state. E. I. Marukovich, A. M. Branovitsky, Y. S. Na, J. H. Lee, K. Y. Choi. // Materials \& Design, Elsevier.-2006.Vol. 27, No. 10, p. 1016-1026.

[4] Guryev M. A. Hardening of cast parts by surface alloying.
[Text] / M. A. Guriev, O. A. Vlasova, A. M. Guryev. // Modern Metal Materials and Technologies, Proceedings of the International Scientific and Technical Conference, St. Petersburg, Polytechnical University, 2009, p. 163-166.

[5] Rasulov F. R. Increase in the quality of the material of the surface layer of castings of cast iron products. Baku, Mechanics-Mechanical Engineering, 2005, p. 41-42.

[6] Baeva L. S., Medko E. K. Increasing the durability of machine parts by applying multicomponent protective diffusion coatings. The invention in mechanical engineering, 2000, №3, p. 15-17.

[7] Zhukov A. A., Silman G. I., Froltsov M. S. Wear-resistant castings from complex-alloyed white cast irons. M., Mechanical Engineering, 2007, 88 p.

[8] Lakhtin Y. M. Material Science / Y. M. Lakhtin, V. P. Leontieva / M., Publishing House Alliance, 2009, 528 p.

[9] Kidalov N. A. Surface alloying of the casting "link of the caterpillar" in the form. / Kidalov N. A., Grebnev Y. V., Zharkova V. F. // Foundry production, 2015, No. 5, p. 9-10.

[10] Rasulov F. R., Babayev. A. I. Increase in corrosion resistance of cast iron castings by surface alloying. Moscow, Bulletin of Mechanical Engineering, No. 12, 2016, p. 75-77.

[11] Research of abrasive-corrosion resistance of chromium cast irons. / L. Y. Kozlov, E. V. Rozhkova, A. A. Kirillov. Proceedings of the III International Scientific and Practical Conference "Progressive Foundry Technologies", 2005, p. 5561 . 